\title{
Lotus leaf inspired robust superhydrophobic coating from strawberry-like Janus particles
}

\author{
Haili Yang, Fuxin Liang, Ying Chen, Qian Wang, Xiaozhong Qu and Zhenzhong Yang
}

We demonstrate a one-step approach toward the large-scale fabrication of robust superhydrophobic coatings using strawberry-like hemispherical Janus particles. Hemispherical Janus particles are capable of self-organizing into a layer on substrates. Nanoscale roughness on the hydrophobic hemispherical side determines the superhydrophobic performance. The imidazolin group on the hydrophilic flat side determines the coating strength by covalent binding onto substrates via cations initiating the crosslinking of the intermediate epoxy resins. The coating can tolerate organic solvents and high water flushing speeds. If the hydrophobic side is smooth, then the coating is highly adhesive to water. This procedure can fabricate unique coatings on a diverse range of substrates with varied compositions and shapes.

NPG Asia Materials (2015) 7, e176; doi:10.1038/am.2015.33; published online 17 April 2015

\section{INTRODUCTION}

Microsized papillae with nanoscale roughness determine the superhydrophobic performance of a lotus leaf surface with a large contact angle and low sliding angle for water. ${ }^{1-5}$ Lotus leaf effect coatings are self-cleaning, promising in both fundamental research and practical applications. Facile and general methods must be generated for the large-scale fabrication of these robust coatings. Traditional methods of fabrication include lithography, etching, plasma treatment and electrospinning. ${ }^{6,7}$ Etching is restricted to special materials. Plasma treatment requires special facilities. The coatings produced by electrospinning are weak. Therefore, using particles to form superhydrophobic coatings on complex shapes is attractive. However, additional post-modification with hydrophobic compounds is required to create the hydrophobic performance. Inspired by the nanoscale coarse structure of micropapillae onto a lotus leaf surface, we design twofaceted Janus particles with a nanoscale coarse hydrophobic face on one side and hydrophilic face on the other. A robust superhydrophobic coating can be easily fabricated using Janus particles, which are strongly bound onto substrates via the crosslinking of the epoxy resin intermediate layer after the Janus particles are self-organized onto that layer.

Janus materials with two different compositions compartmentalized onto the corresponding sides have been developed rapidly and have diverse potential practical applications. ${ }^{8-11}$ Amphiphilic Janus particles can serve as solid surfactants to act as an anchor at an oil/water interface. Rather than the random orientation of homogeneous particles at a Pickering emulsion interface, Janus particles adopt a preferential standing orientation. Therefore, the hydrophobic and hydrophilic sides orient toward the oil and water phases, respectively. ${ }^{12,13}$ Similarly, Janus particles can adopt a preferential standing orientation at a substrate surface, providing a one-step method to fabricate hydrophobic coatings. Although polymeric Janus particles have been used to fabricate superhydrophobic coatings, the effect arises from the aggregation of particles rather than from individual particles. Furthermore, the polymeric coating is weak and breaks when exposed to solvents. ${ }^{14}$ A smooth silica composite Janus particle is used to develop a water-repellent coating on a fiber surface. ${ }^{15}$ This coating is not superhydrophobic. For the best performance, water droplets start to roll off the surface above a tilt angle of $10^{\circ}$. Fabricating a robust superhydrophobic coating with a tilt angle below $5^{\circ}$ remains challenging.

Herein we propose a straightforward approach for producing a lotus leaf mimetic superhydrophobic coating with strawberry-like Janus particles (Scheme 1). The hydrophobic hemispherical side is coarse at the nanoscale. To enhance the adhesion strength, reactive hydrophilic groups (such as imidazolin groups) are introduced onto the flat side, which can catalyze the curing of epoxy resins at room temperature. A thin layer of liquid epoxy resin (E51) is previously coated onto the substrates (for example, glass; a). After the water evaporates from the aqueous dispersion of the Janus particles, the Janus particles can self-organize into an ordered layer (b). The selforganization is dynamically favored by the hemispherical shape with one flat side. The hydrophobic side directs toward the air, whereas the hydrophilic side remains emerged within the epoxy resin layer. After the cationic crosslinking of the epoxy resin initiated by the imidazolin group, the particles are covalently bound onto the substrates (c). Therefore, a lotus leaf mimetic robust superhydrophobic coating is fabricated. 
a

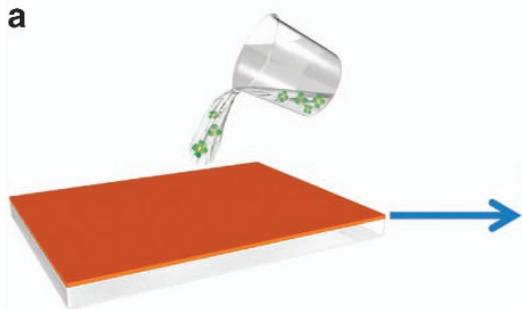

b

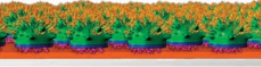

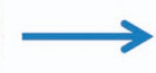

C

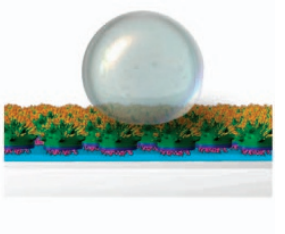

Scheme 1 Fabrication of the lotus leaf mimetic robust superhydrophobic coating. (a) Spraying an aqueous dispersion of the strawberry-like Janus particles onto the substrate with a thin layer of epoxy resin. (b) Janus particles self-orientate into a particle layer after water evaporation; the coarse hydrophobic side directs toward the air and the hydrophilic side is imbedded in the epoxy resin. (c) The cationic crosslinking of the epoxy resin produces a strong bond between the particles and the substrate, forming a robust lotus leaf mimetic superhydrophobic coating.

\section{EXPERIMENTAL PROCEDURE}

\section{Synthesis of Janus particles}

The Janus colloidosomes and particles were synthesized as follows. In total, $10 \mathrm{ml}$ of $10 \mathrm{wt} \%$ hydrolyzed styrene-maleic anhydride copolymer solution and a given amount of Tween- 80 were dissolved in $150 \mathrm{ml}$ of water. A desired amount of $2 \mathrm{moll}^{-1}$ aqueous hydrochloric acid was added to the mixture to adjust the $\mathrm{pH}$ to $\sim 2.5$. To form an oil phase, a given amount of tetraethyl orthosilicate (TEOS), $0.54 \mathrm{~g}$ of aminopropyltrimethoxysilane and a given amount of phenyltriethoxysilane were dissolved in $5 \mathrm{ml}$ of toluene and homogeneously mixed under stirring. After the oil phase was added to the aqueous phase, the mixture was homogenized at a speed of 13000 r.p.m. for 5 min forming an oil-in-water emulsion. The emulsion was incubated at $70^{\circ} \mathrm{C}$ for $12 \mathrm{~h}$ to allow the self-organized interfacial sol-gel process to form a silica shell. After the resultant emulsions were cooled to ambient temperature, the samples were centrifuged at 10000 r.p.m. after washing with water three times. With increasing Tween- 80 content, the Janus colloidosomes will become involved in Janus particles.

Polystyrene (PS) grafting onto the amine group-terminated side of the Janus particles

The Janus particles $(0.1 \mathrm{~g})$ were dispersed in $30 \mathrm{ml}$ of dry dichloromethane containing $2 \mathrm{vol} \%$ of triethylamine. In total, $0.6 \mathrm{ml}$ of 2-bromoisobutyryl bromide was added. The mixture was kept at $0{ }^{\circ} \mathrm{C}$ for $0.5 \mathrm{~h}$ and then at room temperature for another $12 \mathrm{~h}$. The atom transfer radical polymerization agent was conjugated selectively onto the amine group-terminated side of the Janus particles. After centrifugation at 10000 r.p.m. for $5 \mathrm{~min}$ and washing with dichloromethane, the atom transfer radical polymerization agent-terminated Janus particles were obtained as a yellow product.

After $0.1 \mathrm{~g}$ of the atom transfer radical polymerization agent-terminated Janus particles, $12 \mathrm{mg}$ of $\mathrm{N}, \mathrm{N}, \mathrm{N}^{\prime}, \mathrm{N}^{\prime \prime}, \mathrm{N}^{\prime \prime}$-Pentamethyldiethylenetriamine (PMDETA), $1 \mathrm{~g}$ of styrene (St) and $8 \mathrm{ml}$ of toluene were mixed under stirring, the mixture was degassed using three cycles of a freeze pump thaw. In a frozen state, $8 \mathrm{mg}$ of $\mathrm{CuBr}$ was added under the protection of a nitrogen flow. The system was subjected to another freeze pump thaw and sealed under vacuum. After the polymerization at $90^{\circ} \mathrm{C}$ for $12 \mathrm{~h}$, the system was exposed to air to terminate the polymerization. The Janus composite particles were purified after five cycles of centrifugation in toluene and methanol. The PS-grafted Janus composite particles were then obtained.

Imidazolin group grafted on the flat side of the Janus particles In total, $0.1 \mathrm{~g}$ of the PS-grafted Janus composite particle was dispersed in $100 \mathrm{ml}$ of ethanol under stirring. Then, $0.05 \mathrm{~g}$ of triethoxy-3-(2-imidazolin1 -yl) propylsilane was dropped into the dispersion. After the mixture was stirred at $60^{\circ} \mathrm{C}$ for $12 \mathrm{~h}$, the system was cooled to room temperature. The Janus particles were isolated by centrifugation at 6000 r.p.m. After discarding the supernatant, the sediment was re-dispersed in toluene and centrifuged again. The modified Janus particles were achieved.

\section{Fabrication of robust superhydrophobic coating}

In total, $0.5 \mathrm{~g}$ of liquid epoxy resin E-51 was dissolved in $10 \mathrm{ml}$ of acetone. A thin layer of epoxy resin was formed on the glass substrate by spin coating.
An aqueous dispersion of the PS-grafted Janus composite particles was sprayed and kept at room temperature for varied times to ensure a complete crosslinking. After sonication to remove unbound Janus particles, the composite coating was achieved. The contact angles and tilt angles of the water droplets were measured.

\section{RESULTS AND DISCUSSION}

Strawberry-like Janus particles are synthesized by a self-organized solgel process at a patchy emulsion interface. ${ }^{16,17}$ An oil-in-water emulsion forms in the presence of binary surfactants of hydrolyzed styrene-maleic anhydride copolymer and polyoxyethylenesorbitan monooleate (Tween-80; for example, at $0.3 \mathrm{~g}$ ). Tween- 80 is added to induce a phase separation to create patchy domains at the emulsion interface (Supplementary Figure S1). ${ }^{18,19}$ The oil phase is composed of toluene and three silanes: TEOS, aminopropyltrimethoxysilane and phenyltriethoxysilane. A desired amount of $2 \mathrm{~mol}^{-1}$ aqueous hydrochloric acid is added to adjust the $\mathrm{pH}$ to $\sim 2.5$. Under stirring, the emulsion is incubated at $70{ }^{\circ} \mathrm{C}$ for $12 \mathrm{~h}$ for the sol-gel process.

The shell of the hollow sphere is composed of hemispherical particles that protrude outward (Supplementary Figure S2). The particles are connected. Interstitial voids are noted between the particles. The hollow spheres appear as a type of colloidosome with a particle-stacking shell. The colloidosome is stable in organic solvents. The exterior side of the particles is coarse, whereas the interior side is smooth (Figure 1a). Trisodium citrate-capped Au nanoparticles are used to label the particles through a strong interaction with the amine group-terminated regions. The nanoparticles are exclusively absorbed onto the exterior coarse side, whereas the interior side remains smooth (Figure 1b). This absorption indicates that the exterior side is terminated with amine groups. The colloidosome is dispersible in water rather than in hexane, implying that the exterior surface of the colloidosome (thus the particle) is hydrophilic. An example using oil hexane-containing hydrophobic CdSe/ZnS quantum dots shows that these nanomaterials can be captured inside the cavity from the aqueous surroundings (Supplementary Figure S3a). After drying, the $\mathrm{CdSe} / \mathrm{ZnS}$ quantum dots individually precipitate onto the interior surface of the colloidosome (Supplementary Figure S3b). This result implies that the interior surface is hydrophobic, developed from the terminal phenyl group. The wettability of the exterior surface and interior surface is different. The colloidosome is Janus. Tween- 80 has a key role in creating the transverse perforation across the shell. In the absence of Tween-80, a smooth shell forms (Supplementary Figure S4). ${ }^{16}$ When increasing the Tween- 80 content (from 0.5 to $0.8 \mathrm{~g}$ ), the interstitial voids between the particles become larger, whereas their connection becomes weaker (Figure 1c). The exterior side of the particles becomes coarser. Upon slight ultrasonication, the colloidosome is easily disintegrated into individual Janus particles. At $1.0 \mathrm{~g}$ of 
a

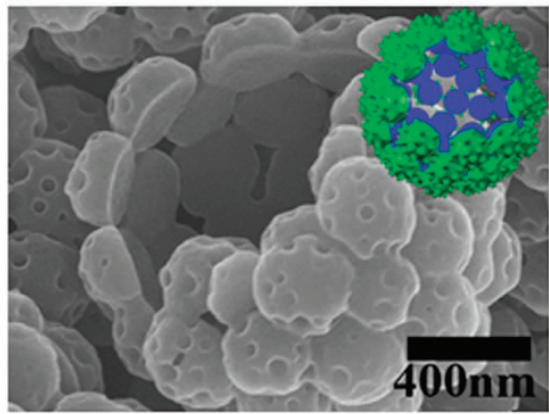

C

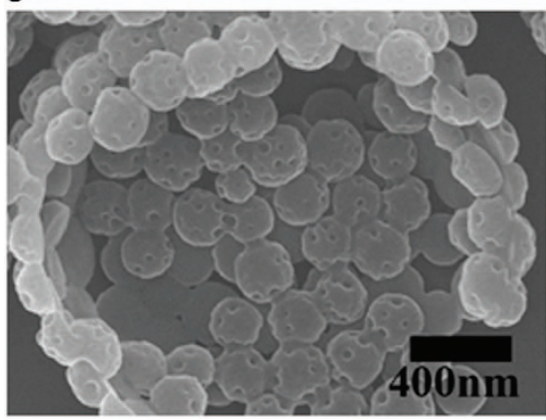

b

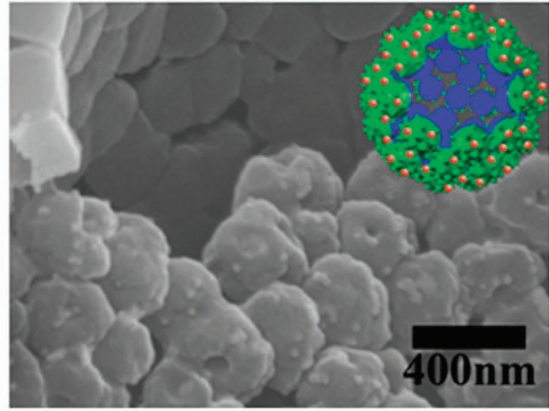

d

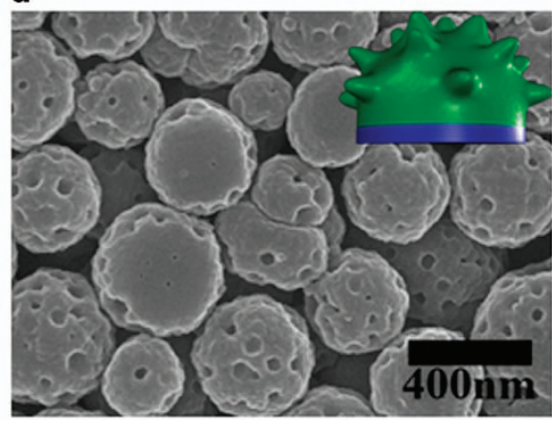

Figure 1 Scanning electron microscopy (SEM) images of the representative colloidosome before (a) and after (b) being labeled with trisodium citrate-capped Au nanoparticles(the inset displays schematic structure); the colloidosome is synthesized at $0.3 \mathrm{~g}$ of Tween-80. (c) SEM image of the colloidosome synthesized at $0.8 \mathrm{~g}$ of Tween-80. (d) Strawberry-like Janus hemispherical particles synthesized at $1.0 \mathrm{~g}$ of Tween-80. Hydrolyzed styrene-maleic anhydride and TEOS are fixed at 1.0 and $0.5 \mathrm{~g}$, respectively.

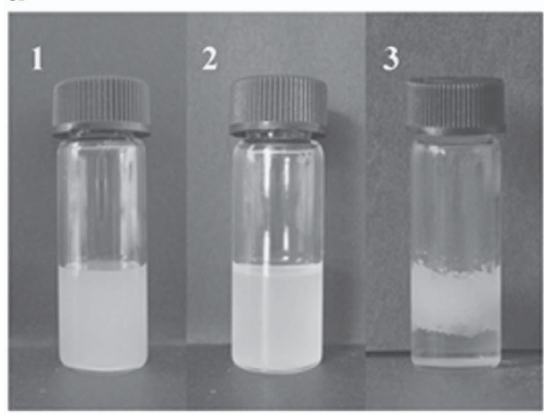

c

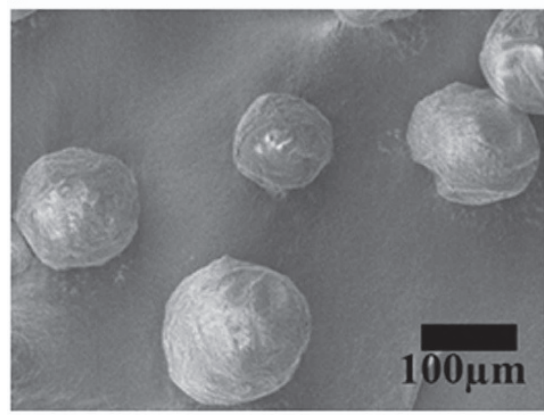

b

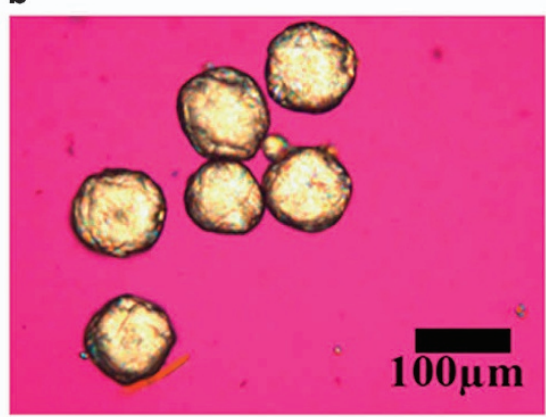

d

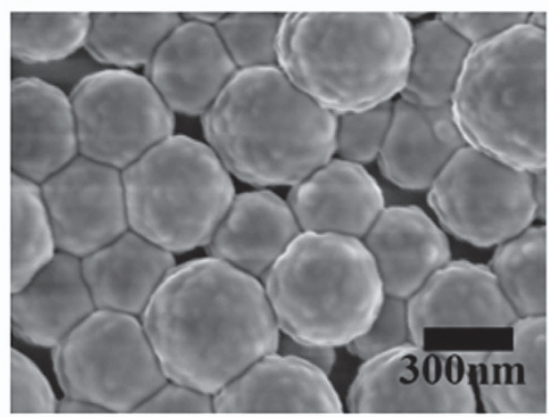

Figure 2 Performance of the Janus particles. (a) Optical images of the Janus particle dispersed in water (1), toluene (2) and (3) the frozen paraffin $\left(\mathrm{Tm}=52-54^{\circ} \mathrm{C}\right.$ )-in-water emulsion stabilized with Janus particles. (b) A polarizing optical microscopy image and (c) scanning electron microscopy (SEM) image of the frozen paraffin emulsion droplets. (d) A magnified SEM image of the Janus particles armoring the surface of the frozen paraffin sphere, all of the hemispherical coarse sides of the particles face toward the aqueous phase. 
a

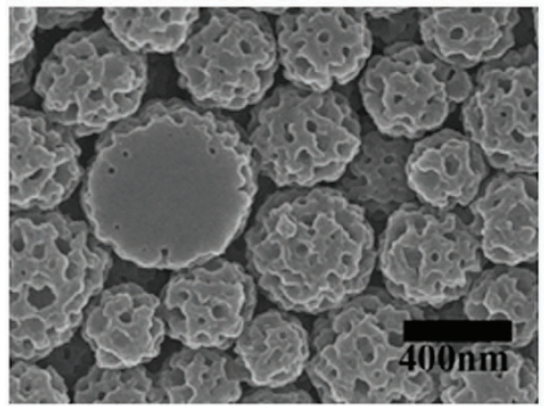

C

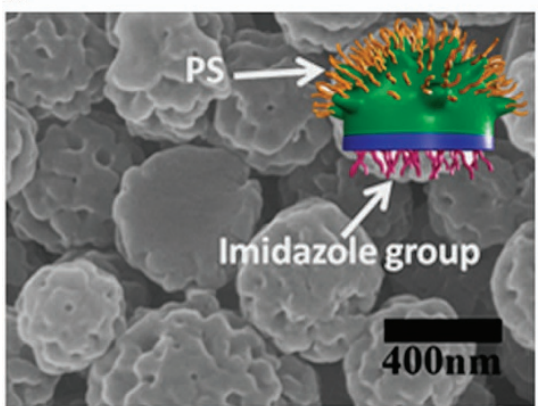

b

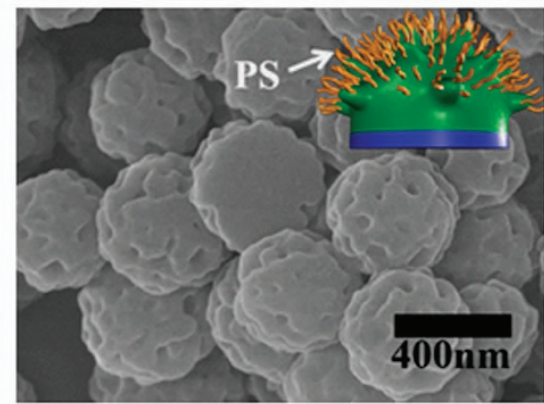

d

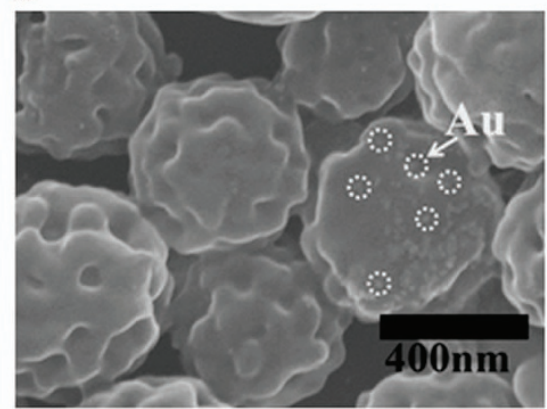

Figure 3 Scanning electron microscopy images of representative Janus particles: (a) Janus particles synthesized using tetraethyl orthosilicate and aminopropyltrimethoxysilane at $2.0 \mathrm{~g}$ of Tween-80; (b) Janus composite particles after grafting PS onto the coarse side(the inset displays the illustrative structure); (c) Janus composite particle after modification of the flat side with trietyoxy-3-(2-imidazolin-1-yl) propylsilane (the inset displays the illustrative structure); and (d) Janus particles after being labeled with the trisodium citrate-capped Au nanoparticles on the modified flat side. PS, polystyrene.

a

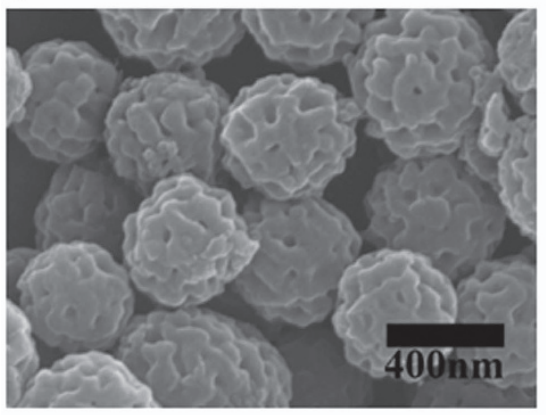

C

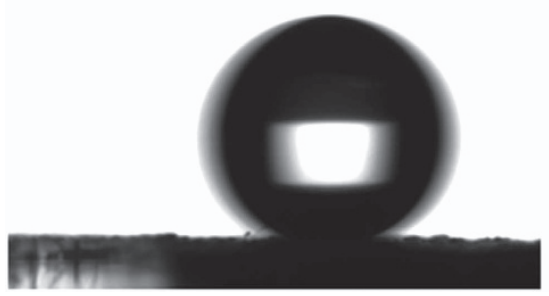

b

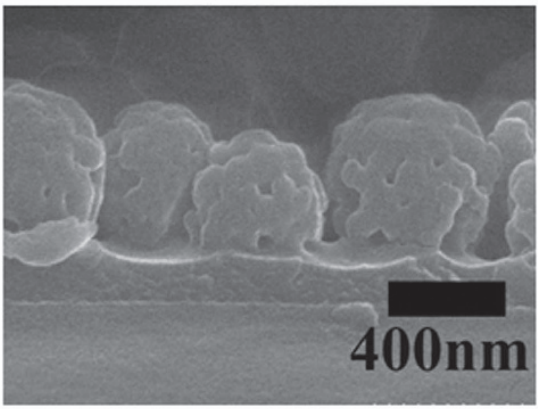

d

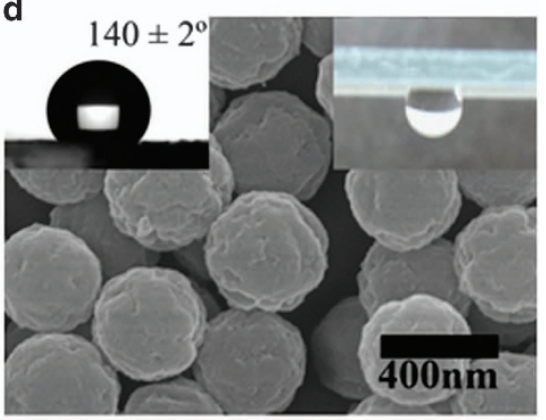

Figure 4 Scanning electron microscopy (SEM) images of the coating of the strawberry-like Janus hemispherical particle. (a) Top and (b) side views. (c) Water droplet contour on the coating. (d) SEM image of the coating of the smooth Janus hemispherical particle; the inset displays a water droplet contour on the coating (left) and after the substrate is inverted (right).

Tween-80, the connection disappears, and strawberry-like Janus hemispherical particles are synthesized (Figure 1d). The diameter of the particles is $\sim 300 \mathrm{~nm}$.
The morphological evolution of the Janus colloidosome during the sol-gel process is monitored. At an early stage $(\sim 10 \mathrm{~min})$, the hollow sphere is weak and collapses after drying. Concaves are noted within 
the shell (Supplementary Figure S5a). In comparison, the shell is smooth without any concaves in the absence of Tween-80. As the sol-gel process progresses ( $180 \mathrm{~min})$, several mosaic discs appear on the shell (Supplementary Figure S5b). The disc and the concave are comparable in size, implying that the discs may grow from the concaves. With further growth ( $240 \mathrm{~min})$, the discs start to protrude outward from the exterior side (Supplementary Figure S5c). Eventually, hemispherical particles are achieved. The exterior surface of the particles becomes coarse (Supplementary Figure S5d). A magnified image shows that the exterior surface of the as-synthesized particles is covered with a material (Supplementary Figure S6a). After washing the exterior surface, several dents remain (Supplementary Figure S6b). Fourier transform infrared spectroscopy results indicate that the material is Tween-80 (Supplementary Figure S7). Tween-80 micelles are formed, which can adhere onto the exterior surface of the discs through a specific interaction. Because Tween-80 is more hydrophilic, an outward convex tension difference can be assumed along the exterior surface of the discs. The protrusion from the exterior surface that forms the hemispherical shape is facilitated under this tension as the sol-gel process progresses. At higher Tween- 80 contents $(2.0 \mathrm{~g})$, the exterior side of the Janus particles becomes coarser (Supplementary Figure S8); the interior side remains smooth. Both the height and cross-section diameter of the Janus particles increase with increasing TEOS content (Supplementary Figure S9).

Similar to Tween-80 (hydrophilic lypophilic balance $($ HLB) $=15$ ), other more hydrophilic surfactants (for example, Tween-20 (HLB = 16.7) and Pluronic F127) are suitable to form Janus colloidosomes. The corresponding Janus particles are achieved at higher surfactant contents (Supplementary Figure S10). By contrast, when more hydrophobic surfactants (for example, sorbitanoleate (Span-80; HLB = 4.3)) are used, smooth hollow spheres form (Supplementary Figure S11). Neither Janus colloidosomes nor Janus particles can be achieved. This result is understandable because no phase separation occurs to form patches at the emulsion interface. In addition to nonionic cosurfactants, other ionic surfactants including SDS and cetyl trimethylammonium bromide are also investigated. No Janus colloidosomes or particles form; no patches are created because the ionic groups are strongly repulsive at the interface. Sufficiently high HLB value of the nonionic co-surfactants is required to form the patchy interface of an emulsion and therefore to form the Janus colloidosomes or particles.

The Janus particles are amphiphilic and well dispersible in water and toluene (Figures 2a1 and a2, respectively). They can serve as a solid emulsifier to stabilize oil-in-water emulsions (Figure 2a3). The emulsion droplets are 20-200 $\mu \mathrm{m}$ in diameter (Figure 2b). Similarly, a paraffin-in-water emulsion forms at high temperatures. After the paraffin core is solidified at room temperature, the orientation of the Janus particles is frozen at the interface (Figure 2c). The paraffin core surface is covered with a single layer of Janus particles. The hydrophilic amine group-terminated coarse hemispherical sides of the particles direct toward the external aqueous phase (Figure 2d).

This approach is universal to synthesize Janus particles with tunable composition, even with other silanes. As an example, when TEOS and aminopropyltrimethoxysilane are mixed in the oil phase, corresponding silica Janus particles form (Figure 3a). The exterior side is coarse and terminated with amine groups. The particles are only dispersible in water rather than in cyclohexane. After selective conjugation of 2-bromoisobutyryl bromide onto the amine group-terminated side (Supplementary Figure S12), polymers (for example, a hydrophobic PS) are grafted by atom transfer radical polymerization. ${ }^{20}$ The coarse microstructure is preserved (Figure $3 \mathrm{~b}$ ). The graftings with 2-bromoisobutyryl bromide and PS are confirmed in the Fourier transform infrared spectroscopy spectra (Supplementary Figure S13). After grafting with PS, the coarse side loses the capability to absorb Au nanoparticles (Supplementary Figure S14), implying the absence of the amine group. The PS-grafted particles become well dispersible both in water and in cyclohexane. Because the flat side contains the $\mathrm{Si}-\mathrm{OH}$ group, the imidazolin group can be introduced by the silane modification (Figure 3c). Trisodium citrate-capped Au nanoparticles are exclusively absorbed on the modified flat side (Figure $3 \mathrm{~d}$ ). In comparison, no Au nanoparticles are found on the unmodified flat side (Supplementary Figure S14). At lower contents of Tween-80, smooth Janus particles are synthesized (Supplementary Figure S15).

The aqueous dispersion of the Janus particles is easily sprayed onto a representative glass substrate. To form a robust coating, a thin layer of liquid epoxy resin (E51) is previously coated onto the substrate. When the Janus particle dispersion is drying on the E51-coating layer, the Janus particles self-organize into a layer. The imidazolin group on the smooth flat side can initiate the curing of E- 51 by cationic polymerization at ambient temperatures. The composite coating is fabricated. The coarse sides of all Janus particles direct toward the air (Figure 4a). A fracture surface image of the coating reveals that the smooth side is embedded within the E51 coating. Because the particles are covalently bound on the coating via crosslinked E51, the coating is sufficiently robust to tolerate solvents and high water flushing. The composite coating can mimic microstructure of lotus leaves with nanoscale coarse micropapillae on the surface. The contact angle of water droplets exceeds $155^{\circ}$. Water droplets can easily role off the coating with a tilt angle below $2^{\circ}$. Therefore, the coating is superhydrophobic. To emphasize the determinative role of the nanoscale roughness in the superhydrophobic performance, another Janus particle (Supplementary Figure S15) with a smooth hydrophobic hemispherical side is used to form the coating. Although the contact angle of water droplets exceeds $140^{\circ}$, the water droplets are strongly pinned onto the coating. These droplets are preserved even after the substrate is inverted by $180^{\circ}$. The coating is highly adhesive to water. This finding is consistent with a previous report on the coating using smooth Janus spherical particles. ${ }^{15}$ In our approach, the hemispherical shape and the imidazolin group of the Janus particles favor an easier dynamic self-orientation and more robust coating.

\section{CONCLUSION}

In summary, we have proposed a straightforward and universal way to fabricate a lotus-leaf superhydrophobic coating using strawberry-like Janus hemispherical particles. The coating is robust, tolerating solvents and rapid water flushing. The nanoscale roughness on the hydrophobic side determines the superhydrophobic performance. The reactive imidazolin group on the flat side favors an easier crosslinking of the epoxy resin intermediate layer, strongly linking the particles with substrates. The coating procedure is easy and general, allowing for the fabrication of unique coatings on many substrates with varied composition and shape. If the hydrophobic side of the Janus particles is smooth, then the coating is highly adhesive to water. This method is expected to produce a responsive superhydrophobic coating when the functional polymers are grafted onto the coarse side.

\section{CONFLICT OF INTEREST}

The authors declare no conflict of interest.

\section{ACKNOWLEDGEMENTS}

This work was supported by the MOST of China (2012CB933200) and the NSF of China (51233007 and 51173191). 
Author contributions: Zhenzhong Yang proposed the project; Zhenzhong Yang and Fuxin Liang designed this work; Haili Yang synthesized the materials and measured the microstructures and properties. All authors participated in the analysis of the experimental data and discussion of the results.

1 Wenzel, R. N. Resistance of solid surfaces to wetting by water. Ind. Eng. Chem. Res. 28, 988-994 (1936).

2 Cassie, A. B. D., Baxter, S. Wettability of porous surfaces. Trans. Faraday Soc. 40 546-551 (1944).

3 Barthlott, W., Neinhuis, C. The effect of ethylene on GTP binding in extracts from pea epicotyls. Planta 201, 1-8 (1997).

4 Neinhuis, C., Barthlott, W. Characterization and distribution of water-repellent, selfcleaning plant surfaces. Ann. Bot. 79, 667-677 (1997).

5 Feng, L., Li, S. H., Li, Y. S., Li, H. J., Zhang, L. J., Zhai, J., Song, Y. L., Liu, B. Q., Jiang, L., Zhu, D. B. Superhydrophobic surfaces: from natural to artificial. Adv. Mater. 14, 1857-1860 (2002)

6 Ma, M. L., Hill, R. M. Superhydrophobic surfaces. Curr. Opin. Colloid Interface Sci. 11, 193-202 (2006)

7 Zhang, X., Shi, F., Niu, J., Jiang, Y. G., Wang, Z. Q. Superhydrophobic surfaces: from structural control to functional application. J. Mater. Chem. 18, 621-633 (2008).

$8 \mathrm{Hu}$, J., Zhou, S. X., Sun, Y. Y., Fang, X. S., Wu, L. M. Fabrication, properties and applications of Janus particles. Chem. Soc. Rev. 41, 4356-4378 (2012).

9 Walther, A., Müller, A. H. E. Janus particles: synthesis, self-assembly, physical properties and applications. Chem. Rev. 113, 5194-5261 (2013).

10 Liang, F. X., Zhang, C. L., Yang, Z. Z. Rational design and synthesis of Janus composites. Adv. Mater. 26, 6944-6949 (2014).

11 Jiang, S., Granick, S. Janus particle synthesis, self-assembly and applications, RSC Cambridge, UK, (2012).

12 Binks, B. P., Lumsdon, S. O. Pickering emulsions stabilized by monodisperse latex particles: effects of particle size. Langmuir 17, 4540-4547 (2001).
13 Binks, B. P., Fletcher, P. D. I. Particles adsorbed at the oil-water interface: a theoretical comparison between spheres of uniform wettability and "Janus" particles. Langmuir 17 4708-4710 (2001).

14 Synytska, A., Khanum, R., Ionov, L., Cherif, C., Bellmann, C. Water-repellent textile via decorating fibers with amphiphilic Janus particles. ACS Appl. Mater. Interfaces 3 1216-1220 (2011).

15 Berger, S., Lonov, L., Synytska, A. Engineering of ultra-hydrophobic functional coatings using controlled aggregation of bicomponent core/shell Janus particles. Adv. Funct. Mater. 21, 2338-2344 (2011).

16 Liang, F. X., Liu, J. G., Zhang, C. L., Qu, X. Z., Li, J. L., Yang, Z. Z. Janus hollow spheres by emulsion interfacial self-assembled sol-gel process. Chem. Commun. 47, 1231-1233 (2011).

17 Chen, Y., Yang, H. L., Zhang, C. L., Wang, Q., Qu, X. Z., Li, J. L., Liang, F. X., Yang, Z. $Z$. Janus cages of bilayered polymer-inorganic composites. Macromolecules 46, 4126-4130 (2013).

18 Sknepnek, R., Vernizzi, G., Cruz, M. O. Buckling of multicomponent elastic shells with line tension. Soft Matter 8, 636-644 (2012).

19 Kamio, E., Yonemura, S., Ono, T., Yoshizawa, H. Microcapsules with macroholes prepared by the competitive adsorption of surfactants on emulsion droplet surfaces. Langmuir 24, 13287-13298 (2008).

20 Yang, H. L., Liang, F. X., Wang, X., Chen, Y., Zhang, C. L., Wang, Q., Qu, X. Z., Li, J. L., Wu, D. C., Yang, Z. Z. Responsive Janus composite nanosheets. Macromolecules 6 , 2754-2759 (2013)

(c) (i) This work is licensed under a Creative Commons Attribution 4.0 International License. The images or other third party material in this article are included in the article's Creative Commons license, unless indicated otherwise in the credit line; if the material is not included under the Creative Commons license, users will need to obtain permission from the license holder to reproduce the material. To view a copy of this license, visit http:// creativecommons.org/licenses/by/4.0/

Supplementary Information accompanies the paper on the NPG Asia Materials website (http://www.nature.com/am) 